\title{
Effect of Safety Leadership on Employee's Safety Attitude and Safety Behavior
}

\author{
안전리더십이 근로자의 \\ 안전태도와 안전행동에 미치는 영향
}

\author{
Kuk Hoan Wee ${ }^{1}$, Kwang Kyu Kim², Seong Rok Chang ${ }^{3}$, Won Seok Bang ${ }^{4}$, Sun Hwa Kim ${ }^{5}$ \\ 위국환 ${ }^{1}$, 김광규 $^{2}$, 장성록 ${ }^{3}$, 방원석 $^{4}$, 김선화 $^{5}$ \\ ${ }^{1}$ Professor, GSIS Department, Pusan National University, Korea, wkh64@pusan.ac.kr \\ ${ }^{2}$ Professor, Department of Counselling Social Work, Canada Christian College, Korea, \\ kimgyoo@daum.net \\ ${ }^{3}$ Professor, Department of Safety Engineering, Pukyong National University, Korea, \\ srchang@pknu.ac.kr \\ ${ }^{4}$ Professor, Department of Entrepreneurship Research Institute, Gyeongsang National University, \\ Korea,bangws@gnu.ac.kr \\ ${ }^{5}$ Professor, Department of Judicial Affairs Business, Korea Maritime and Ocean University, Korea, \\ ksh66@kmou.ac.kr
}

Corresponding Author: Sun Hwa Kim

\begin{abstract}
The number of workers who got involved in industrial disaster gradually increased year by year. The purpose of this study is to identify the relationships between manager's safety leadership, safety attitudes and safety behaviors. A survey of plant workers and plant partner staff was conducted and the SPSS 21.0 program and Amos 21.0 were used for analysis. The results of the study revealed the following: First, sub-dimensions of safety leadership have a significant positive effect on personal safety attitude, except for trust relationship. Second, it has been shown that sub-dimensions of safety leadership have significant positive effects on personal safety behavior except for trust relationships. Based on the research results, as safety leadership increases, personal safety attitudes and personal safety behaviors enhance. Therefore, the researchers present safety self-coaching as the important role of personal safety attitude and behavior. Also, some academic and practical implications based on the research results were presented. In addition, there is a common task of reducing safety accidents by increasing the motivation and commitment of wokers to safety. However, until now, the safety behavior of workers has been forced only in the direction without any motivating factors. In this regard, the study suggests that safety self-coaching as one of the leadership style is meaningful in the industrial field.
\end{abstract}

Keywords: Safety Attitude, Safety Behavior, Safety Leadership, Trust

요약: 산업재해를 당한 근로자의 수는 해마다 점차 증가하고 있다. 이 연구의 목적은 관리자 의 안전 리더십, 안전 태도 및 안전 행동 간의 관계를 조사 분석하고자 한다. 발전도 근로자

Received: August 07, 2021; $1^{\text {st }}$ Review Result: September 23, 2021; $2^{\text {nd }}$ Review Result: November 14, 2021 Accepted: December 31, 2021 
를 대상으로 설문조사를 실시하였으며 분석은 SPSS 21.0 프로그램과 Amos 21.0을 이용하였다. 연구결과에 따르면 첫째, 안전 리더십의 하위 차원은 신뢰 관계를 제외하고 개인의 안전 태 도에 유의한 정 $(+)$ 의 영향을 미치는 것으로 나타났다. 둘째, 안전 리더십의 하위 차원은 신뢰 관계를 제외하고 개인의 안전 행동에 유의한 긍정적인 영향을 미치는 것으로 나타났다. 즉, 리더의 안전 리더십이 높을수록 개인의 안전 태도와 개인의 안전 행동이 향상된다. 따라서 리더의 안전셀프코칭을 근로자 개인의 안전 태도와 행동의 중요한 역할로 제시한다. 연구결 과를 바탕으로 학문적, 실질적 시사점을 제시한다, 또한, 근무자의 안전에 대한 동기와 의지 를 높여 안전사고를 줄이는 것이 공통 과제이다. 그러나 지금까지 근로자의 안전행동은 아무 런 동기 요인 없이 강요되어 왔다. 그런 점에서 우리는 안전리더십 스타일의 하나로 안전셀 프코칭을 통한 지도가 산업 현장에서 의미가 있다고 제안한다.

핵심어: 안전태도, 안전행동, 안전리더십, 신뢰

\section{1. 서론}

고용노동부 통계조사[1]에 따르면 2020년도 산업재해를 당한 근로자 수는 총 근로자 $18,725,160$ 명 중 109,242 명이었다. 그중 사고 사망자는 882 명으로 전년 대비 27 명 $(3.2 \%)$ 증가하였으며 사고 사망률은 $0.46 \%$ 이었다. 2014 년 이후 지속적으로 $0.5 \%$ 대를 유지하고 있으나, 재해율은 $0.49 \%$ 로 전년도 $0.50 \%$ 에 비해 다소 감소하였다. 최근 화력발전소 산업재해로는 2018년 충남 태안화력발전소에 근무하던 비정규직 $\mathrm{K}$ 씨가 컨베이어벨트에 끼여 사망하였고[2] 2017년 삼척화력발전소의 $\mathrm{J}$ 씨는 비계 설치 작업 중 추락사고로 사망하였다[3].

이런 산업재해의 원인을 살펴보면, 국가적인 측면에서는 안전정책에 관한 늦장 대응과 사회적인 측면에서는 외주화에 따른 산업현장의 안전관리와 안전의식의 부족이라 할 수 있다. 그리고 개인적인 측면에서는 근로자의 불완전한 행동이 증가하고 있기 때문이다. 이러한 불안전한 행동은 근로자가 안전수칙을 준수하고자 하는 의지가 없거나 지키기 어려운 여건에 기인한 것으로 볼 수 있다. 산업현장 안전을 위해 안전설비와 제도적인 분야는 그동안 많은 진전이 있었다. 하지만 안전수준을 한 단계 더 높이려면 안전태도와 행동을 변화시키는 안전리더십에 대한 연구가 더 필요하다. 뿐만 아니라 산업재해의 근본적인 예방을 위해서는 근로자들의 불안전한 행동을 안전한 행동으로 변화시키는 방안에 대한 연구가 지속적으로 진행되어야 한다[4].

기업에서는 산업재해 예방을 위해 개인의 안전태도나 행동 변화에 대한 연구가 활발히 진행되어 왔다. 안전행동의 중요성에 관한 연구 중 윤영만[5]은 행동기반 안전 적용사례를 통한 효과성을 분석하였고, 이주영[6]은 안전학습이 기업의 안전분위기 및 근로자의 안전행동에 영향을 준다는 것을 밝혔다. 이찬[7]은 도시철도조직에서 근로자가 안전행동을 하고 사고를 줄이기 위한 방안으로 안전친화적 기업문화가 영향을 준다고 밝혔다. 또한 전우명과 임춘성[8]은 시설관리기업의 안전문화가 안전행동과 경영성과에 긍정적인 영향을 준다고 밝혔고, 김민지 등[9]은 심리적 안정이 창의성 및 학습 행동에 정적인 영향을 미친다고 밝혔다. 이원준과 이은영[10]은 국내 항공산업을 중심으로 서비스기업의 지향적 리더십이 안전관리 행동에 영향을 미친다고 보고하였다. 재해방지를 위해서는 안전태도도 큰 영향을 미친다. 김성애 등[11]은 방사선 안전관리를 치위생과 학생들에게 교육한 결과 방사선 방어에 대한 지식과 태도에 많은 변화가 
생긴다는 것을 보고하였다. 임대성[12]은 국가 대형 안전사고 발생 전후를 비교하여 산업위생 분야 종사자들이 사회 안전 의식에 많은 변화가 있다는 것을 검증하였다. 또한 김혜연[13]은 의료기관 인증제 인식정도가 간호사의 환자안전태도에 많은 영향을 미친다고 보고하였다.

본 연구에서는 개인의 안전행동과 태도를 변화시키는 주요 요인 간의 상관관계를 검증해 보고 그 결과에 따라 발전소 현장에 적용 가능한 효과적인 안전관리 방안을 제시하고자 한다. 이를 위해 안전 리더십을 독립변수로 설정하고, 개인안전 행동변화 및 개인안전 태도변화를 종속변수로 설정하여 연구를 진행하였다.

\section{2.연구방법}

\section{1 연구모형}

본 연구의 목적은 안전종사들의 안전리더십이 안전태도와 행동에 미치는 영향의 관계를 파악하고자 한다. 이에 다음과 같이 [그림 1]과 같이 연구모형을 설계하였다.

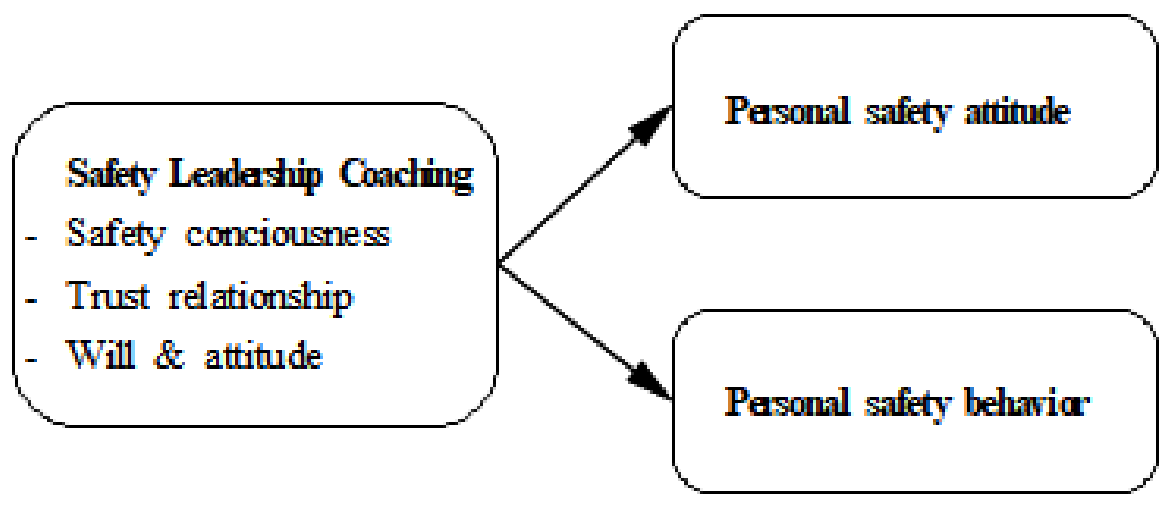

[그림 1] 연구모형

[Fig 1] Research Hypothesis

\section{2 연구가설 설정}

안전리더십, 개인안전 태도, 개인안전 행동 간의 관계를 선행연구를 살펴본 결과 안전리더십과 개인안전 태도 간에 관계성이 있었으며 안전리더십과 개인안전 행동 간에도 유의미한 관계성이 있었다. 이러한 선행연구를 근거로 삼아 다음과 같은 가설을 설정하고자 한다.

$<$ 가설 1>. 안전리더십은 안전태도에 정 $(+)$ 의 영향을 미칠 것이다.

$<$ 가설 1-1>. 안전의식은 안전태도에 정 $(+)$ 의 영향을 미칠 것이다.

$<$ 가설 1-2>. 신뢰관계는 안전태도에 정 $(+)$ 의 영향을 미칠 것이다.

$<$ 가설 1-3>. 의지, 태도는 안전태도에 정 $(+)$ 의 영향을 미칠 것이다.

$<$ 가설 2>. 안전리더십은 안전행동에 정 $(+)$ 의 영향을 미칠 것이다.

$<$ 가설 2-1>. 안전의식은 안전행동에 정 $(+)$ 의 영향을 미칠 것이다.

$<$ 가설 2-2>. 신뢰관계는 안전행동에 정 $(+)$ 의 영향을 미칠 것이다.

$<$ 가설 2-3>. 의지, 태도는 안전행동에 정 $(+)$ 의 영향을 미칠 것이다. 


\section{3 설문지 구성}

본 연구를 위해 설문지는 문헌조사와 선행연구를 토대로 구성하였다. 조사 대상자의 인구통계학적 특성을 포함하여 총 40항목으로 [표 1]과 같이 구성하였으며, 설문 문항은 리커트(Likerrt) 5점 척도를 사용하여 측정하였다.

\section{[표 1] 설문지 구성}

[Table 1] Structure of Survey Sheet

\begin{tabular}{|c|c|c|c|}
\hline $\begin{array}{l}\text { Measuring } \\
\text { variable }\end{array}$ & $\begin{array}{c}\text { Number of } \\
\text { questions }\end{array}$ & Previous study & Measuring scale \\
\hline Safety Leadership & 18 & $\begin{array}{l}\text { Kyeong-hoon Lee, Safety Leader's Impact on the Safety Culture of } \\
\text { Workplaces, Ph.D. at Chosun University [2012] }\end{array}$ & Likert 5 \\
\hline $\begin{array}{l}\text { Safety behavior } \\
\text { and attitude }\end{array}$ & 18 & $\begin{array}{l}\text { Kyeong hoon Lee, A Study on the Effect of Safety Leadership of Site } \\
\text { Managers on the Safety Culture of Workplaces, Journal of the Ph.D. } \\
\text { in the Graduate School of Chosun University [2012] }\end{array}$ & Likert 5 \\
\hline $\begin{array}{l}\text { Demographic } \\
\text { Characteristics }\end{array}$ & 4 & $\begin{array}{c}\text { Gender, Age(Over 20,30,40,50,60s) } \\
\text { Position (Employee, manager, section chief, deputy director, } \\
\text { department head, higher than head) } \\
\text { Working period(Less than } 5 \text { years, } 5-10,10-15,15-20 \text {,More than } 20 \\
\text { years) }\end{array}$ & \\
\hline Total & 40 & & \\
\hline
\end{tabular}

\section{4 표본선정과 자료수집의 방법}

발전소 근무직원 및 발전소 협력사 직원 가운데 작업자, 공사감독, 기타 근무자 등 약 340 명을 대상으로 설문조사를 하였다. 그중에서 성실하지 못한 설문지를 제외한 329명의 설문조사 결과를 대상으로 분석은 SPSS PASW Statistics 21.0 프로그램과 Amos를 사용하여 통계분석을 실시하였다.

\section{3. 연구방법}

\section{1 인구통계학적 분석}

먼저 성별을 살펴보면, 남자는 275 명으로 $83.6 \%$, 여자는 54 명으로 $16.4 \%$ 으로 나타났다. 연령을 살펴보면, 20대는 62명(18.8\%), 30대는 136명(41.3\%), 40대는 68명(20.7\%), 50대는 62 명 $(18.8 \%), 60$ 대 이상 1 명 $(0.3 \%)$ 으로 나타났다. 직급을 살펴보면, 사원은 $34.0 \%(112$ 명), 주임/대리는 21.6\%(71명), 과장/차장은 39.8\%(131명), 부장 $4.6 \%(15$ 명)으로 나타났다. 근무 년을 살펴보면, 5년 미만은 36.2\%으로 119 명, 5 10년 미만은 $22.2 \%$ 으로 73 명, 10 15년 미만은 $11.9 \%$ 으로 39명, 15 20년 미만은 5.5\%으로 18명, 20년 이상 $24.3 \%$ 으로 80 명으로 나타났다. 
[표 2] 인구통계학적 분석결과

[Table 2] Demographic Analysis

\begin{tabular}{|c|c|c|c|}
\hline Variable & Item & Frequency & Fraction $(\%)$ \\
\hline \multirow{2}{*}{ Gender } & Male & 275 & 83.6 \\
\hline & Female & 54 & 16.4 \\
\hline \multirow{5}{*}{ Range of Age } & $20 \mathrm{~s}$ & 62 & 18.8 \\
\hline & $30 \mathrm{~s}$ & 136 & 41.3 \\
\hline & $40 \mathrm{~s}$ & 68 & 20.7 \\
\hline & $50 \mathrm{~s}$ & 62 & 18.8 \\
\hline & More than 60 & 1 & 0.3 \\
\hline \multirow{4}{*}{ Position } & Employee & 112 & 34.0 \\
\hline & Chief & 71 & 21.6 \\
\hline & Section Chief & 131 & 39.8 \\
\hline & Head of department & 15 & 4.6 \\
\hline \multirow{5}{*}{$\begin{array}{c}\text { Working } \\
\text { period }\end{array}$} & Less than 5 years & 119 & 36.2 \\
\hline & $5 \sim 10$ years & 73 & 22.2 \\
\hline & $10 \sim 15$ years & 39 & 11.9 \\
\hline & 15 20 years & 18 & 5.5 \\
\hline & More than 20 years & 80 & 24.3 \\
\hline
\end{tabular}

\section{2 신뢰도와 타당도 분석}

안전의식에 대한 신뢰도의 값은 0.926 으로 매우 높은 신뢰도를 보이고 있고, 신뢰관계에 대한 신뢰도의 값도 0.913으로 매우 높게 나타났으며 의지태도에 대한 신뢰도의 값도 0.943 으로 매우 높게 나타났다. 개인안전태도에 대한 신뢰도의 값은 0.952로 매우 높게 나타났으며, 개인안전행동에 대한 신뢰도의 값도 0.896 으로 높게 나타났다[표 3].

\section{[표 3] 신뢰성 분석결과}

[Table 3] Results of Reliability Analysis of Factors

\begin{tabular}{c|c|c}
\hline Variable & Number of questions & Cronbach`s Alpha \\
\hline \hline Safety consciousness & 7 & .926 \\
\hline Trust relationship & 4 & .913 \\
\hline Will \& Attitude & 7 & .943 \\
\hline Personal safety attitude & 11 & .952 \\
\hline Personal safety behavior & 6 & .896 \\
\hline
\end{tabular}

\section{3 확인적 요인분석}

먼저 구조방정식 모형분석을 하기 전에 각 잠재변인을 구성하는 관측 변인이 타당하게 구성되어 있는지를 살펴보기 위해 확인적 요인분석(Confirmatory factor analysis: CFA)을 실시하였다. 측정모형의 적합도 지수를 개선하기 위해 측정항목의 표준화 계수가 0.7 
이하로 나타난 안전행동 $\mathrm{BC6}$ 를 제거하였다.

본 연구에서는 적합도 평가 지수의 기준이 확립된 CFI(Comparative Fit Index), TLI(TuckerLewis Indes), RMSEA(Root-Mean Squre Error of Approxi -mation)를 통해 모형의 적합도를 평가하였다. 증분적합지수 중 $\mathrm{CFI}$ 값과 $\mathrm{TLI}$ 값은 높으면 높을수록 모형의 적합도가 좋다고 볼 수 있는데 대략 0.9 이상이면 좋은 적합도라고 판단한다.

반면 RMSEA 값은 작을수록 적합도가 좋은 것으로 판단한다. RMSEA < .05이면 매우 좋은 적합도, RMSEA < .08이면 좋은 적합도, RMSEA < .10이면 보통 적합도, RMSEA $>.10$ 이면 나쁜 적합도라고 할 수 있다[14][15].

증분적합지수인 TLI는 기준치인 0.923 의 값을 나타냈으며, CFI도 기준치에 해당하는 0.9 보다 높은 값인 0.923 을 나타냈다. RMSEA(절대적합지수)는 기준치인 .10보다 낮게 나타나 확인적 요인분석 모형은 적합하다고 판단할 수 있다. 물론 통계학적으로 적합도의 절대기준치가 아직까지 정해진 것은 없지만 통상학적으로 위 기준들은 적합한 기준치라고 할 수 있다[표 4 참조].

[표 4] 확인적요인 적합성분석 결과

[Table 4] Results of Confirmatory Factor Analysis

\begin{tabular}{|c|c|c|c|c|c|c|c|}
\hline \multirow[b]{2}{*}{$\chi^{2}$} & \multirow[b]{2}{*}{$d f$} & \multirow[b]{2}{*}{$p$} & \multirow[b]{2}{*}{$T L I$} & \multirow[b]{2}{*}{$C F I$} & \multicolumn{3}{|c|}{ RMSEA } \\
\hline & & & & & Value & $\begin{array}{l}\text { Lower } \\
\text { Bound }\end{array}$ & $\begin{array}{l}\text { Upper } \\
\text { Bound }\end{array}$ \\
\hline 1776.145 & 839 & .000 & .923 & .923 & .058 & .055 & .062 \\
\hline
\end{tabular}

확인적 요인분석을 실시할 때 각 관측변인이 잠재변인을 잘 반영하고 있는지를 파악하기 위해 관측변인들의 요인 부하량을 확인하였다. 확인한 결과 모든 경로계수가 유의미한 것으로 나타났기 때문에 관측변인들이 해당 잠재변인을 잘 반영하고 있다고 판단되었다. 한편 표준화 경로계수는 0.5 이상이어서 개념 타당도를 만족시키는 것으로 판단되었다[표 5].

[표 5] 확인적요인 분석결과

[Table 5] Results of Confimatory Factor Analysis

\begin{tabular}{|c|c|c|c|c|c|c|}
\hline \multicolumn{3}{|c|}{ Path } & \multirow[t]{2}{*}{ S.E. } & \multirow{2}{*}{$\begin{array}{c}\text { Estimate } \\
1.000\end{array}$} & \multirow[t]{2}{*}{ C.R. } & \multirow[t]{2}{*}{$\mathrm{P}$} \\
\hline SS7 & $<-$ & SS & & & & \\
\hline SS6 & $<-$ & SS & .051 & 1.019 & 19.867 & $* * *$ \\
\hline SS5 & $<-$ & SS & .047 & .970 & 20.712 & $* * *$ \\
\hline $\mathrm{SS} 4$ & $<-$ & $\mathrm{SS}$ & .053 & .914 & 17.226 & $* * *$ \\
\hline SS3 & $<--$ & SS & .068 & 1.125 & 16.518 & $* * *$ \\
\hline $\mathrm{SS} 2$ & $<-$ & SS & .048 & .906 & 19.077 & $* * *$ \\
\hline SS1 & $<-$ & SS & .052 & .889 & 17.219 & $* * *$ \\
\hline TR4 & $<-$ & TR & .053 & .931 & 17.469 & $* * *$ \\
\hline TR3 & $<-$ & TR & & 1.000 & & \\
\hline TR2 & $<-$ & TR & .060 & 1.179 & 19.518 & $* * *$ \\
\hline TR1 & $<--$ & TR & .058 & 1.208 & 20.694 & $* * *$ \\
\hline
\end{tabular}




\begin{tabular}{|c|c|c|c|c|c|c|}
\hline WI7 & $<--$ & WI & & 1.000 & & \\
\hline WI6 & $<-$ & WI & .057 & 1.145 & 20.118 & $* * *$ \\
\hline WI5 & $<--$ & WI & .051 & 1.047 & 20.559 & $* * *$ \\
\hline WI4 & $<-$ & WI & .060 & 1.214 & 20.096 & $* * *$ \\
\hline WI3 & $<-$ & WI & .064 & 1.230 & 19.086 & $* * *$ \\
\hline WI2 & $<-$ & WI & .059 & 1.260 & 21.495 & $* * *$ \\
\hline WI1 & $<-$ & WI & .051 & .967 & 18.935 & *** \\
\hline $\mathrm{AC7}$ & $<--$ & $\mathrm{AC}$ & & 1.000 & & \\
\hline AC6 & $<-$ & $\mathrm{AC}$ & .059 & 1.084 & 18.293 & *** \\
\hline AC5 & $<-$ & $\mathrm{AC}$ & .056 & 1.038 & 18.396 & $* * *$ \\
\hline AC4 & $<-$ & $\mathrm{AC}$ & .057 & .976 & 17.101 & $* * *$ \\
\hline $\mathrm{AC} 3$ & $<-$ & $\mathrm{AC}$ & .057 & .885 & 15.444 & $* * *$ \\
\hline $\mathrm{AC} 2$ & $<-$ & $\mathrm{AC}$ & .059 & .969 & 16.566 & *** \\
\hline $\mathrm{AC} 1$ & $<-$ & $\mathrm{AC}$ & .057 & .931 & 16.280 & *** \\
\hline AC8 & $<-$ & $\mathrm{AC}$ & .059 & 1.001 & 17.095 & $* * *$ \\
\hline AC9 & $<-$ & $\mathrm{AC}$ & .059 & .985 & 16.720 & $* * *$ \\
\hline $\mathrm{AC} 10$ & $<-$ & $\mathrm{AC}$ & .060 & 1.062 & 17.846 & $* * *$ \\
\hline $\mathrm{AC} 11$ & $<--$ & $\mathrm{AC}$ & .060 & 1.047 & 17.427 & $* * *$ \\
\hline $\mathrm{BC} 7$ & $<-$ & $\mathrm{BC}$ & & 1.000 & & \\
\hline BC5 & $<-$ & $\mathrm{BC}$ & .058 & .979 & 17.000 & $* * *$ \\
\hline BC4 & $<-$ & $\mathrm{BC}$ & .055 & .931 & 16.961 & $* * *$ \\
\hline BC3 & $<--$ & $\mathrm{BC}$ & .053 & .919 & 17.271 & $* * *$ \\
\hline $\mathrm{BC} 2$ & $<--$ & $\mathrm{BC}$ & .054 & .870 & 16.019 & $* * *$ \\
\hline $\mathrm{BC} 1$ & $<-$ & $\mathrm{BC}$ & .050 & .913 & 18.421 & $* * *$ \\
\hline
\end{tabular}

Explanation of abbreviation- Coaching leadership of safety (CL), Safety consciousness (SS), Trust Relationship (TR), Will and Intention (WI)- Attitudinal changes of personal safety (AC), Behavioral changes of personal safety (BC)

\section{4 상관관계분석}

변수들의 상관관계를 보기 위하여 Pearson 상관분석을 사용하였다. 안전의식과 신뢰관계를 살펴보면, 상관계수가 0.750 으로 유의미한 양의 상관관계를 보였고 $(\mathrm{p}<0.05)$, 안전의식과 의지태도를 살펴보면 상관계수가 0.799 으로 유의미한 양의 상관관계를 보였다 $(\mathrm{p}<0.05)$. 그리고 안전의식과 개인안전행동을 살펴보면 상관계수가 0.719 으로 유의미한 양의 상관관계를 보였고 $(\mathrm{p}<0.05)$, 안전의식과 개인안전태도를 살펴보면, 상관계수가 0.763 으로 유의미한 양의 상관관계를 보였다 $(\mathrm{p}<0.05)[$ 표 6].

[표 6] 상관관계 분석결과

[Table 6] Result of Correlation of Factors

\begin{tabular}{c|c|c|c|c|c}
\hline Variable & 1 & 2 & 3 & 4 & 5 \\
\hline \hline $\begin{array}{c}\text { Safety consciousness } \\
(1)\end{array}$ & 1 & & & & \\
\hline $\begin{array}{c}\text { Trust Relationship } \\
(2)\end{array}$ & $0.317^{* *}$ & 1 & & & \\
\hline
\end{tabular}




\begin{tabular}{c|c|c|c|c|c}
\hline $\begin{array}{c}\text { Will \& Attitude } \\
(3)\end{array}$ & $0.313^{* *}$ & $0.283^{* *}$ & 1 & 1 & \\
\hline $\begin{array}{c}\text { Personal safety } \\
\text { attitude } \\
(4)\end{array}$ & $0.293^{* *}$ & $0.333^{* *}$ & $0.548^{* *}$ & $0.154^{* *}$ & 1 \\
\hline $\begin{array}{c}\text { Personal safety } \\
\text { behavio } \\
(5)\end{array}$ & $0.159^{* *}$ & $0.165^{* *}$ & $0.291^{* *}$ & 1 \\
\hline
\end{tabular}

$* * \mathrm{p}<0.01$

안전의식과 신뢰관계를 살펴보면, 상관계수가 0.317 로 통계적으로 유의미한 양의 상관관계를 보였으며 $(\mathrm{p}<0.01)$, 안전의식과 의지태도를 살펴보면 상관계수가 0.313 으로 통계적으로 유의미한 양의 상관관계를 나타냈다 $(\mathrm{p}<0.01)$. 안전의식과 안전태도 및 안전행동 간의 상관계수가 각각 $0.293,0.159$ 으로 통계적으로 유의미한 양의 상관관계를 보이는 것으로 확인했다 $(\mathrm{p}<0.01)$. 또한, 신뢰관계와 의지태도, 안전태도, 안전행동 간의 상관계수가 각각 $0.293,0.333,0.163$ 으로 통계적으로 유의미한 양의 상관관계를 보였다 $(\mathrm{p}<0.01)$. 의지태도와 안전태도, 안전행동 간의 상관계수가 각각 $0.548,0.291$ 으로 통계적으로 유의미한 양의 상관관계를 나타냈다 $(\mathrm{p}<0.01)$. 끝으로, 개인안전행동과 개인안전태도를 살펴보면, 상관계수가 0.154 로 통계적으로 유의미한 양의 상관관계를 보였다 $(\mathrm{p}<0.01)$.

\section{5 수렴타당도}

수렴타당도는 동일한 개념을 서로 다른 방법을 사용하여 측정했을 경우, 각 측정 결과 간의 상관관계가 높고 낮음을 분석하여 타당도를 검증하는 방법이다. 이러한 수렴타당도를 검정하기 위한 방법으로 각 변수에 대한 개념 신뢰도(Construct Relibility)와 평균분산 추출값(Average Variance Extracted)을 측정하였다. 수렴타당도를 평가할 때 개념 신뢰도의 값이 0.7 이상, 평균분산 추출값이 0.5 이상이면 수렴타당도가 높다고 판단한다[표 7].

[표 7] 표 제목 수렴타당도 분석결과

[Table 7] Results of Convergent Validity

\begin{tabular}{c|c|c}
\hline Variable & Concept Reliability & AVE \\
\hline \hline Safety consciousness & .964 & .793 \\
\hline Trust Relationship & .942 & .804 \\
\hline Will \& Attitude & .960 & .773 \\
\hline Personal safety behavior & .976 & .889 \\
\hline Personal safety attitude & .959 & .895 \\
\hline
\end{tabular}

\section{4.결과분석}

가설검증결과, 먼저 안전의식이 안전태도에 미치는 영향을 살펴보면 $\quad(\beta=0.179$, $\mathrm{t}=2.138$ )로 유의수준 0.05 에서 통계적으로 유의미한 양의 영향을 미치는 것으로 나타났다. 신뢰관계가 안전태도에 미치는 영향을 살펴보면 (경로계수=0.180, $\mathrm{t}=-1.576$ )로 유의수준 
0.05에서 통계적으로 유의하지 않은 것으로 확인되었다. 의지태도가 안전태도에 미치는 영향을 살펴보면 (경로계수 $-0.115, \mathrm{t}=3.088$ )로 유의수준 0.01 에서 통계적으로 유의미한 양의 영향을 미치는 것으로 나타났다. 또한, 신뢰관계가 안전행동에 미치는 영향을 살펴보면 (경로계수 $=0.268, \mathrm{t}=2.164$ )로 유의수준 0.05 에서 통계적으로 유의미한 영향을 미치는 것으로 확인되었다. 안전의식이 안전행동에 미치는 영향을 살펴보면 (경로계수 $=0.234, \mathrm{t}=0.460$ )로 유의수준 0.05 에서 통계적으로 유의미한 양의 영향을 미치는 것으로 나타났다. 신뢰관계가 안전태도에 미치는 영향을 살펴보면 (경로계수 $=0.043$, $\mathrm{t}=2.158$ )로 유의수준 0.05 에서 통계적으로 유의미한 영향을 미치는 것으로 입증되었다[표 $8]$.

[표 8] 경로분석 결과

[Table 8] Results of Path Analysis

\begin{tabular}{c|c|c|c|c|c}
\hline Path & Path Cofficient & S.E. & t-value. & p-value & Result \\
\hline \hline $\mathrm{SC} \rightarrow \mathrm{AC}$ & .180 & .084 & 2.138 & $.033^{* *}$ & Supported \\
\hline $\mathrm{TR} \rightarrow \mathrm{AC}$ & -.115 & .073 & -1.576 & .115 & Not supported \\
\hline $\mathrm{WI} \rightarrow \mathrm{AC}$ & .268 & .087 & 3.088 & $.002^{* *}$ & Supported \\
\hline $\mathrm{SC} \rightarrow \mathrm{BC}$ & .234 & .108 & 2.164 & $.030^{* *}$ & Supported \\
\hline $\mathrm{TR} \rightarrow \mathrm{BC}$ & .043 & .093 & .460 & .646 & Not supported \\
\hline $\mathrm{WI} \rightarrow \mathrm{BC}$ & .239 & .111 & 2.158 & $.031^{* *}$ & Supported \\
\hline
\end{tabular}

$* \mathrm{p}<0.05 * * \mathrm{p}<0.01$

* Explanation of abbreviation

- Coaching leadership of safety (CL), Safety consciousness (SC), Trust Relationship (TR), Will and Intention (WI)

- Personal safety attitude (AC), Personal safety behavior(BC)

\section{5. 결론}

우리나라는 2018년 이후부터 안전에 대한 사망사고 등의 감소를 위해 다양한 정책을 추진하고 안전 최우선을 표방하고 있지만 안전사고는 여전히 줄어들지 않고 있다. 실제로도 우리 국민의 안전문화 수용성과 안전에 대한 태도와 행동 의식은 과거에 비해 크게 달라지지 않고 있다. 그런데 대부분의 사고는 예측이 불가능하기 때문에 예방이 어렵기는 하지만 전혀 불가능한 것은 아니다. 사고 예방을 위해 안전문화를 강조하는 것은 매우 중요한데 그중에서도 안전리더십은 핵심 요소라고 할 수 있다. 특히 관리자의 안전리더십이 조직의 안전문화나 분위기에 영향을 미친다는 것은 이미 많은 선행연구를 통해 밝혀졌다. 최근 기업들은 재해 예방을 위해 안전행동과 태도의 변화에 안전의식, 신뢰 관계, 의지 및 태도 등을 변화 시켜 안전한 사업장을 만들기 위한 여러 가지 방안들을 구상하는데 많은 노력을 기울이고 있다.

본 연구에서는 안전리더십이 근로자의 안전행동에 영향을 미친다는 것을 확인하고 관리자의 안전리더십을 통해 개인의 안전행동과 태도를 변화시켜 개인의 안전 확보방안을 모색하고자 하였다.

연구가설을 검증한 결과는 다음과 같다.

첫째, 안전리더십이 개인안전 태도 변화에 미치는 영향을 살펴보기 위해 변수 등을 
통제하고 회귀분석을 진행한 결과, 신뢰 관계를 제외하고 안전리더십의 다른 하위요소와 개인안전 태도 변화 간에 통계적으로 유의한 정적 영향을 미치는 것으로 나타났다. 둘째, 안전리더십이 개인안전 행동 변화에 미치는 영향을 살펴보기 위해 변수 등을 통제하고 회귀분석을 진행한 결과, 신뢰 관계를 제외하고 안전 리더십의 다른 하위요소와 개인안전 행동 변화 간에 통계적으로 유의한 정적 영향을 미치는 것으로 나타났다. 이상의 연구가설 검증 결과를 종합해보면 안전리더십이 높아질수록 이로 인한 개인안전 태도 및 개인안전 행동에 정 $(+)$ 의 영향을 주는 것으로 조사되었다. 이는 결국 안전리더십이 높아질수록 개인안전 태도와 개인안전 행동에 긍정적인 영향을 미치는 것으로 볼 수 있다.

따라서 본 연구자는 근로자가 안전지속 의도를 높일 수 있는 방안으로 안전코칭리더십을 제안한다. 이러한 안전코칭리더십은 안전의식, 신뢰 관계, 안전의지 및 태도를 구성원들에게 모델로 제시함으로써 구성원들의 안전 태도와 행동에 긍정적 영향을 미친다. 결과적으로 안전코칭리더십은 구성원들의 태도와 행동 변화를 통해 조직의 안전문화를 구축하는 데 중요한 역할을 하는 선행요인임을 실증적으로 제시한다.

추가로 향후 연구 방향으로 안전코칭리더십을 통한 스스로 실시하는 안전셀프코칭을 제안하고자 한다. 안전셀프코칭은 근로자가 자율학습 도구 등을 활용하여 스스로 안전한 태도 및 행동을 지속적해서 유지하도록 도와야 한다. 자율학습 도구로는 휴대폰 $\mathrm{APP}$ 를 사용해 주어진 질문에 스스로 응답하여 안전에 대한 자각을 통해 안전의식과 태도, 행동을 유발되게 하는 것이 필요하다.

오늘날의 기업조직은 안전에 관한 관심과 정책이 매우 중요하고 이러한 정책에 구성원 등을 참여 시켜 성과를 창출해야 하는 변화가 요구되고 있다. 또한 직원들의 안전에 대한 동기유발과 몰입을 높여 안전사고를 줄여나가야 하는 공통적인 과제를 안고 있다. 그런데 지금까지 근로자의 안전행동은 동기 요인 없이 방향성만 강요되어 왔다. 이런 점에서 근로자 스스로 문제를 해결하고 이끌어나갈 수 있게 하는 안전셀프코칭은 산업 현장에서 적극적으로 활용이 가능하다는 점에서 의의가 있다고 할 수 있다.

본 논문의 연구 결과를 바탕으로 학문적, 실무적 시사점을 제공하고 있지만, 한계점으로 안전리더십과 안전태도 및 행동 간 관련성이 있는 것으로 확인되었으나, 실제로 그러한가에 대해서는 더욱더 충분한 토의와 연구가 필요하다고 사료된다.

\section{References}

[1] https://eboard.moel.go.kr, Jun 20 (2021)

[2] www.gidream.com/news/articleView.html, Jun 30 (2021)

[3] J. K. You, A Study on the Establishment of Owner-led Safety Management System in the Construction of Thermal Power Plant, Seoul Science and Technology University, Master's thesis, pp.14-18, (2018)

[4] J. M. Yang, Y. G. Kwon, Effect of Behavior Based Safety Program on Safety Behavior, Safety Climate and its Satisfaction, Journal of the Korean Society of Safety, (2018), Vol.33, No.5, pp.109-119, DOI: 10.14346/JKOSOS.2018.33.5.109

[5] Y. M. Yoon, Analysis through the Application Case of Behavior-Based Safety (BBS), Seoul Science and Technology University, Master's thesis, pp.41-42, (2017)

[6] J. Y. Lee, The Influence of Learning among Safety Culture Factors on Safety Environment and Safety Behavior of Workers, Hansung University, Master's thesis, pp.54-55, (2017) 
[7] C. Lee, The Influence of the Urban Railway Organization's Friendly Corporate Culture on Safety Tendency and Safety Behavior and Thinking, Seoul Science and Technology University, Master's thesis, pp.44-45, (2015)

[8] W. M. Jeon, C. S. Lim, A Study on the Impact of Safety Culture on Safety Behavior and Management Performance of the Facility Management Company, Korean Management Consulting Review, (2018), Vol.18, No.2, pp.195-215.

[9] M. J. Kim, J. M. Kim, The Effects of Diversity and Psychological Safety on Creativity and Learning Behavior of Project Teams in Large Corporations - Focusing on the Mediating Effect of Creativity, Journal of Corporate Education and Talent Research, (2014), Vol.16, No.2, pp.57-88, UCI : G704-002126.2014.16.2.005

[10] W. J. Lee, E. Y. Lee, The Convergence and Global Management of the Aviation, Tourism and Service Industries: A Study on the Influence of Task-Oriented Leadership in the Safety Management Behavior and Perceptual Performance of Service Companies - Focusing on the domestic aviation industry, AIK Spring Conference Proceeding, Aviation Management Society of Korea, pp.61-71, (2004), May 29, Korea.

[11] S. A. Kim, J. Y. Lee, S. H. Hwang, M. S. Cho, J. H. Park, N. Y. Jung, B. C. Yu, Knowledge and attitude change towards radiation protection after radiation safety management education in dental hygiene students, Journal of Korean society of Dental Hygiene, (2015), Vol.15, No.1, pp.101-109, DOI: 10.13065/jksdh.2015.15.01.101

[12] D. S. Lim, A Study on the Change of Social Safety Consciousness of Workers in Industrial Hygiene: comparative Study on the Major National Safety Accidents Before and After, Hansung University, Ph.D dissertation, pp.80-85, (2016)

[13] H. Y. Kim, The Effect of the Perception of Medical Institutions on the Patient Safety Attitude of Nurses, Gachon University, Master's thesis, pp.58-59, (2016)

[14] J. P. Woo, Structural equation model concept and understanding, Hannarae Publishing, pp.167-172, (2016)

[15] M. W. Browne, R. Cudeck, Alternative Ways of Assessing Model Fit, Sociological Methods \& Research, (1992), Vol.21, No.2, pp.230-258, DOI: https://doi.org/10.1177/0049124192021002005 